\title{
Correspondence
}

\section{Couch persons}

Sir: Private practice psychiatry is worth scrutinising. Patients slip into a medical office, see the psychiatrist, reappear 50 minutes later and disappear into the crowd. Millions of these contractual relationships are made worldwide each year without any scientific evaluation. Most patients go with depressive illnesses, anxiety states or domestic crises.

Previously we described a venture combining psychotherapy and pharmacology within psychiatry (Eastwood \& Shneiderman, 1994). This is a short account of the first year of operations. Sixty patients were referred from psychotherapy for a pharmacology opinion. The patients were middle everything: age, class, income and management. The findings may apply everywhere.

While selected, the population is interesting. The sexes were equally represented, in their mid-40s, and mostly married with children. Women were more likely to be single or divorced. Most men and one-third of women had university education. Most were employed. They had major depression, dysthymia and, then, anxiety states. They were, by nature, frequently fussy, rigid, moody, anxious and shy. Women had worried more than men. Most had friends and interests. They were social drinkers and a few smoked. Most had family histories of depression, alcoholism or suicide. Stressors were marriage, health, work, finances and children. Few needed admittance to hospital. Parasuicide was not an issue. Patients received psychotherapy and SSRI drugs.

Outcome? The antidepressants had proven effectiveness and, assuming good compliance. efficiency. Psychotherapy obviously had not been tested in the same way (WPA Dysthymia Working Group, 1995). Twenty per cent dropped out, $5 \%$ needed admittance to hospital, with the remainder a mishmash. The psychotherapist (GS) referred everybody for pharmacology to the doctor at the Clarke Institute (RE). Some found the toing and froing between doctors, understanding protocols and waiting for efficacy, tedious. Nevertheless, 46 , or about $75 \%$, continued with the twin treatments: 13 recovered and 33 improved. Outcome, decided between the two doctors, was mostly the opinion of the psychotherapist, since these were his patients.

We combined medication and psychotherapy approaches so that affectively disturbed patients, when calm and cheerful on medication, might deal with life. The patients, busy and functional people, appeared surprisingly well, following treatment. This result, however, will remain unacceptable, to 'evidence based medicine'. Nevertheless, a recent review said, "He (Archie Cochrane) obviously recognized the limited impact of randomized controlled trial (RCT) methodology in psychiatry and one longs to know what adaptations of the method he might have proposed to accommodate these areas such as psychotherapy which do not readily fit the classical design." (Shanks, 1994).

Patients like these are never going to agree to RCTs. Yet depression is so common and so untreated. Which comes first, strategy or incontrovertible scientific proof?

EASTwOOD, R. \& Schneiderman, G. (1994) Couch fellows. Lancet, 343, 131-132.

SHANKS. J. (1994) Books reconsidered. Effectiveness and Efficiency. (A. L. Cochrane). British Journal of Psychiatry, 165, 702-704.

WPA DYSTHYMIA WORKING GROUP (1995) Dysthyma in clinical practice. British Journal of Psychiatry, 166, 174-183.

ROBIN EASTWOOD, Clarke Institute and GERALD SCHNEIDERMAN, Hospital for Sick Children, University of Toronto, Canada M5T 1R8

\section{Arrangements for MRCPsych examinations}

Sir: I was interested to read the letter from Dr P. Sen (Psychiatric Bulletin, June 1995, 19, 380) concerning the way our examinations for the MRCPsych are conducted. There has, of course, been a long tradition in British medicine of the 'sudden death' examination where candidates are given the result shortly after the last viva.

The examinations for MRCPsych involve a large number of candidates, are held in many centres and utilise double marking in all but 